\title{
Forame oval patente e acidente vascular cerebral: causa e consequência?
}

\author{
Patent foramen ovale and stroke: cause and consequence?
}

\author{
Ana Verena Silvany Sampaio de Miranda ${ }^{1}$ \\ Claudia Yamada Utagawa
}

1 Discente do Curso de Medicina do Centro Universitário de Volta Redonda.
2 Docente do Curso de Medicina do Centro Universitário de Volta Redonda.

\section{RESUMO}

O forame oval patente (FOP) é um defeito embriológico cardíaco consequente da não fusão dos septum primum e septum secundum do átrio primitivo. Sua incidência é de $25 \%$ na população geral. Na literatura, ele aparece associado a várias patologias, dentre elas, acidente vascular cerebral (AVC), infarto agudo do miocárdio e enxaqueca. 0 objetivo deste trabalho é revisar a literatura sobre os aspectos embriológicos e correlações clínicas do FOP com AVC e as possíveis explicações fisiopatológicas envolvidas nessa associação clínica. Foram utilizados artigos científicos dos bancos de dados do Pubmed, COCHRANE e Scielo entre os anos de 2008 a 2013. As palavras-chaves utilizadas foram "Foramen Ovale, Patent" and "Stroke". Clinicamente o FOP não apresenta sintomas, sendo encontrado mais frequentemente como um achado fortuito em investigação de outros problemas cardíacos ou em eventos de AVC. O FOP foi encontrado em $53 \%$ dos casos estudados de AVC criptogênico, que compreendem $30 \%$ a $40 \%$ de todos os eventos de AVCs. Por conta desses achados, estudos têm atribuído ao FOP uma provável causa etiológica do AVC criptogênico. Uma hipótese relacionada ao mecanismo de interação entre o FOP e o AVC é que o FOP pode atuar como um canal para a embolização paradoxal. Devido à grande prevalência do FOP na população, é necessário, portanto, ficar atento aos sintomas que o paciente apresenta, pois esse defeito septal pode estar associado com o aumento do risco de AVC criptogênico.

\section{PALAVRAS-CHAVE}

Forame oval patente, Acidente vascular cerebral, AVC criptogênico.

\begin{abstract}
The patent foramen ovale (PFO) is a cardiac embryologic defect consequent of no fusion of septum primum and septum secundum of primitive atrium. The PFO incidence is about $25 \%$ of general population. In the literature it has been associated with a lot of pathologies as stroke, myocardial infarction and migraine. The purpose of this study is to review the literature about the embryologic aspects and clinical correlations between PFO and stroke and the possible physiopathologic explanation involved in this clinical association. Scientific articles were searching on PubMed, COCHRANE and Scielo databases from 2008 to 2013. The keywords used were "Foramen Ovale, Patent" and "Stroke". The PFO doesn't present clinical symptoms, which has been found more frequently as a casual finding in an others cardiologic problems or stroke events investigation. The PFO was found in $53 \%$ of the studied cryptogenic stroke cases that include $30 \%$ to $40 \%$ of all stoke events. Due these findings, studies show that PFO can be conferred to a possible etiologic cause to cryptogenic stroke. A related hypothesis for interaction mechanism between PFO and stroke is that PFO can be act as a channel to the paradoxal embolization. Due the large prevalence of the PFO in the population, it is necessary, therefore, to have attention to the symptoms presents in the patient because this septal defect can be related of the cryptogenic stroke risk increase.
\end{abstract}

\section{KEYWORDS}

Patent foramen ovale, stroke, cryptogenic stroke.

\section{Como você deve citar?}

MIRANDA, Ana Verena Silvany Sampaio de; UTAGAWA, Claudia Yamada. Forame oval patente e acidente vascular cerebral: causa e consequência?. Cadernos UniFOA, Volta Redonda, n. 26, p. 131-136, dez. 2014. 


\section{INTRODUÇÃO}

$\mathrm{Na}$ vida intrauterina, o forame oval é essencial, pois permite que a maior parte do sangue mais oxigenado oriundo da placenta atravesse o septo interatrial para atingir diretamente a circulação sistêmica, sem passar pelos pulmões, que nessa fase do desenvolvimento encontram-se colabados (ESTEVES et al., 2010). Após a primeira inspiração, ao nascimento, os pulmões expandem-se e ocorre uma elevação do retorno sanguíneo para o átrio esquerdo, fazendo com que as duas estruturas embrionárias que formam o septo interatrial, o septum primum e o septum secundum fundem-se e, dessa forma, ocluem o forame oval. Em alguns indivíduos, tais estruturas não se fundem, resultando na persistência do forame. Com o aumento da pressão intratorácica e a persistência do orifício, o fluxo sanguíneo do átrio esquerdo passa para o átrio direito, caracterizando o forame oval patente (FOP) (URIBE, 2008), considerado, portanto, um defeito do septo atrial.

A prevalência de FOP na população geral é de aproximadamente $25 \%$ segundo Wechsler (2008 apud LANTZ, 2012), de acordo com os dados obtidos com o estudo em autópsias. Essa prevalência também foi encontrada por Homma e Di Tullio, em 2010, em pacientes utilizando ecocardiograma transesofágico. Em outro estudo, com indivíduos com mais de 80 anos de idade, a prevalência encontrada foi de aproximadamente $20 \%$ (CRUZ-GONZÁLEZ et al., 2008).

Na literatura, existem estudos de associação do FOP com diversas patologias, incluindo acidente vascular cerebral (AVC), enxaqueca (Di TULLIO, 2010; OZDEMIR et al., 2008; ANZOLA; MAZZUCCO, 2008), síndrome descompressiva em mergulhadores, embolia periférica incluindo infarto do miocárdio (JOHANSSON; ERIKSSON; DELLBORG, 2009), insuficiência renal e mal de Alzheimer (VIGNA et al., 2008). A comunicação da direita para a esquerda através do FOP no coração pode levar a uma piora significativa dos sintomas em pacientes com doença pulmonar crônica associada com hipóxia, ou apneia do sono (KUTTY; SENGUPTA; KHANDHERIA, 2012).

O objetivo deste artigo é revisar a literatura dos últimos cinco anos sobre os aspectos embriológicos e correlações clínicas do FOP com acidente vascular cerebral e as possíveis explicações fisiopatológicas envolvidas nessa associação clínica.

\section{MATERIAIS E MÉTODOS}

Para a elaboração desta revisão de literatura foram utilizados artigos científicos dos bancos de dados do Pubmed, COCHRANE e Scielo, entre os anos de 2008 a 2013, com os seguintes descritores: "Patent foramen ovale" and "Stroke". Os artigos foram selecionados nos idiomas: inglês, português e espanhol. Foram excluídos os relatos de casos e séries de casos, totalizando 31 artigos: duas meta-análises, oito revisões sistemáticas, duas revisões narrativas e 19 revisões integrativas.

\section{RESULTADOS}

\subsection{Embriologia}

O septo interatrial cardíaco é uma estrutura que divide o átrio primitivo em cavidades direita e esquerda. Iniciado na quinta semana de gestação, o septum primum começa a desenvolver-se a partir do teto da parede do átrio direito, crescendo em direção ao coxim endocárdico. Entre essas duas estruturas, verifica-se a presença de um forame chamado de ostium primum que se oclue após a fusão do septum primum com o coxim endocárdico. Depois da fusão, pequenas perfurações desenvolvem-se 
na porção cefálica do septum primum, que formarão a seguir o ostium secundum. Logo à direita do septum primum, começa a formar uma invaginação na parede atrial, o septum secundum. Esse septo para seu crescimento no final da sétima semana de gestação, deixando uma lacuna posterior e inferior conhecida como forame oval (ROJAS et al., 2010).

Ao nascimento, os orifícios interatriais fecham-se devido a diminuição da pressão pulmonar, e a pressão atrial esquerda excede a direita. Dentro alguns meses de vida, o forame oval fecha a passagem na maioria dos indivíduos. Entretanto, em uma considerável parte da população, o forame oval permanece aberto (THALER; SAVER, 2008).

\subsection{Sinais E Sintomas}

O FOP é clinicamente assintomático, porém o indicador mais comum para sua detecção foi representado por recentes ou remotos eventos de acidentes vasculares cerebrais, somando mais da metade de todos os achados $(54,2 \%)$, seguida de uma descoberta causal durante a realização de um exame de ecocardiografia para outros problemas cardíacos em aproximadamente um terço (35\%). A enxaqueca foi a causa dos restantes $10,5 \%$ do total dos grupos de estudo (FAGGIANO et al., 2012). Por meio de um estudo realizado por Ebrahimi et al., no ano de 2011, encontrou-se uma taxa maior do que $53 \%$ de presença de FOP em casos de AVC criptogênico, ou seja, sem causa conhecida definida, compreendendo $30 \%$ a $40 \%$ de todos os AVCs (PRISTIPINO et al., 2012; GUERCINI et al., 2008).

\subsection{Métodos diagnósticos}

Os procedimentos para detecção do FOP mais comumente usados são: ecocardiograma transesofágico, que investiga e avalia a função ventricular, morfologia e funcionamento das válvulas, além da aurícula esquerda e do septo interatrial (TELMAN et al., 2008; GUPTA et al., 2008); ecocardiograma transtorácico, que não examina o átrio tão bem como o ecocardiograma transesofágico (THALER; SAVER, 2008); e o doppler transcraniano, que detecta sinais microembólicos por meio da injeção de contrastes (WYNGAERT et al., 2008; RUNDEK, 2008).

\subsection{Tratamento}

Em pacientes assintomáticos e sem história médica pregressa de AVC criptogênico, no qual o FOP foi encontrado acidentalmente, nenhum tratamento é recomendado a não ser que o AVC criptogênico silencioso seja diagnosticado depois de estudos de imagem, como a tomografia computadorizada (TC) ou ressonância magnética (RM). Portanto, a primeira prevenção do AVC, seja ela com medicamentos ou com dispositivos feitos para a terapia, não é indicada para pacientes com FOP encontrados de maneira fortuita. Em pacientes com AVC isquêmico criptogênico, a estratégia terapêutica é baseada na avaliação da probabilidade do FOP estar casualmente relacionado ao evento clínico e do risco de recorrência (PRISTIPINO et al., 2012).

$\mathrm{O}$ tratamento medicamentoso tem sido usado em pacientes com AVC e FOP e pode ser feito à base de antitrombóticos, por meio de anticoagulantes orais como a varfarina ou tratamento com agentes antiplaquetários, geralmente aspirina (Di TULLIO, 2010; WECHSLER, 2008). Por meio de uma meta-análise de 15 estudos previamente publicados com pacientes com FOP, a combinação de AVC recorrente, morte ou ataque isquêmico transitório (AIT) com tratamento medicamentoso foi estimado em 4 eventos por 100 indivíduos/ano (ALMEKHLAFI et al., 2009).

Há também o fechamento percutâneo que utiliza uma técnica transcateter para ocluir o septo atrial. Diversos ensaios não randomizados sugerem um potencial benefício no fechamento do FOP em 
pacientes com AVC criptogênico. A eficácia desse tratamento para abolir a comunicação entre os átrios encontra-se nas faixas entre $86 \%$ a 100\% (ONORATO et al., 2008; CAPUTI et al., 2012; CAPUTI et al., 2013).

O fechamento do FOP pela técnica transcateter foi associado com $84 \%$ da redução de eventos neurológicos recorrentes comparados com a administração de medicamentos (AGARWAL et al., 2012).

No geral, os pacientes no estudo do fechamento do FOP tendem a ser mais jovens e possuir alta frequência de eventos tromboembólicos anteriores, enquanto os pacientes no estudo do tratamento medicamentosos tendem a possuir uma alta frequência de fatores de risco comuns para o AVC, como diabetes e fumo (HOMMA \& Di TULLIO, 2010).

O fechamento cirúrgico do FOP, nos dias atuais, é raramente feito. Suas principais indicações são auxiliar nos casos de procedimentos falhos, usando a técnica transcateter ou em indivíduos com hipersensibilidade a alguns dos componentes dos dispositivos - por exemplo, no caso de hipersensibilidade ao níquel (Di TULLIO, 2010; RIGATELLI; CARDAIOLI; CHINAGLIA, 2008).

\section{DISCUSSÃo}

Potenciais mecanismos de associação do AVC com anomalias do septo atrial incluem a embolização paradoxal através do FOP, fibrilação atrial paroxística e formação de trombos dentro da cavidade do FOP (FORCE; MASSABUAU; LARRUE, 2008).

Uma suposta explicação a respeito do mecanismo do AVC é que o FOP pode atuar como um canal para a embolização paradoxal, uma condição na qual o êmbolo de origem venosa entra na circulação sistêmica pelo canal que comunica o átrio direito com o esquerdo, formado pelo FOP, devido a um shunt direito-esquerdo (MATTLE et al., 2010; GROGONO et al., 2012). A embolização paradoxal foi descrita pela primeira vez por Cohnheim, em 1877, quando, então, esse autor utilizou a terminologia para descrever o fenômeno (MATTLE et al., 2010). A existência desse mecanismo é documentada pela detecção ocasional do trombo apresentado em pacientes com casos de embolia (Di TULLIO, 2010).

Como possíveis cofatores associados ao aparecimento de AVC, temos como os mais citados: variantes anatômicos, relacionando o tamanho do FOP com o risco de AVC (GOEL apud HOMMA \& Di TULLIO, 2010). Outro fator associado é o hemodinâmico, como condições que aumentam a pressão do átrio direito, levando a um maior desvio da direita para a esquerda através do FOP e, como consequência, a probabilidade de uma embolia paradoxal (HOMMA \& Di TULLIO, 2010).

Alguns autores, entretanto, questionam o papel do FOP na gênese dos AVC isquêmicos, uma vez que, apesar do FOP estar presente em quase $25 \%$ da população, a embolização paradoxal é considerada um evento relativamente raro. Estudos populacionais estimam que, ao longo de 70 anos de vida, apenas $2 \%$ dos FOP seriam sintomáticos, apresentando-se como AVC (MATTLE et al., 2010).

\section{CONCLUSÃO}

A prevalência do FOP na população geral chama a atenção, por ser de aproximadamente $25 \%$. A maioria dos indivíduos portadores de FOP, entretanto, é assintomático, sendo que o defeito é detectado em investigação para outras patologias. $O$ achado do FOP, entretanto, em mais de $53 \%$ de casos de AVC criptogênico, deve ser um alerta, para que não seja desconsiderada como causa etiológica, uma vez que a embolização paradoxal, apesar de rara, é um evento reconhecido como uma de suas complicações. 
A presença da FOP isolada apresenta baixo risco para AVC isquêmico e, portanto, nenhuma profilaxia primária é recomendada (MATTLE et al., 2010), assim como não é indicado o rastreamento para pacientes assintomáticos. Em pacientes com FOP que já apresentaram quadros isquêmicos, sintomáticos ou não, o tratamento preventivo deve ser considerado, principalmente nos casos de pacientes jovens.

\section{REFERÊNCIAS}

AGARWAL, S. et al. Meta-analysis of transcatheter closure versus medical therapy for patent foramen ovale in prevention of recurrent neurological events after presumed paradoxical embolism. Jacc: cardiovascular interventions, v. 5, n. 7, 2012.

ALMEKHLAFI, M.A. et al. Recurrent cerebral ischemia in medically treated patent foramen ovale: a metaanalysis. Neurology, v. 73, n. 2, p. 89-97, 2009.

ANZOLA, G.P.; MAZZUCCO, S. The patent foramen ovale-migraine connection: a new perspective to demonstrate a causal relation. Neurol Sci, v. 29, p. 15-18, 2008.

CAPUTI, L. et al. Residual Shunt after Patent Foramen Ovale Closure: Preliminary Results from Italian Patent Foramen Ovale Survey. Journal of Stroke and Cerebrovascular Diseases, p. 1-8, 2013.

CAPUTI, L. et al. Italian patent foramen ovale survey (I.P.O.S.): Early results. Perspectives in Medicine, v. 1, p. 236-40, 2012.

CRUZ-GONZÁLEZ, I. et al. Foramen oval permeable: situación actual. Rev. Esp. Cardiol., v. 61, p. 738$51,2008$.

Di TULLIO, M.R. Patent Foramen Ovale: Echocardiographic detection and clinical relevance in stroke. Journal of the American Society of Echocardiography, v. 23, n. 2, 2010.

EBRAHIMI, H.A; MOGHADAM, A.H.; AREDESTANI, E. Evaluation of patent foramen ovale in young adults with cryptogenic stroke. ARYA Atherosclerosis Journal, v. 7, n. 2, 2011.

ESTEVES, V. et al. Oclusão percutânea do forame oval patente com prótese PREMERETM: resultados preliminares da primeira experiência no Brasil. Rev. Bras. Cardiol. Invasiva, v. 18, n. 1, 2010.

FAGGIANO, P. et al. Low cerebrovascular event rate in the subjects with patent foramen ovale and different clinical presentations. International Journal of Cardiology, v.156, p. 47-52, 2012.

FORCE, M.; MASSABUAU, P.; LARRUE, V. Prevalence of atrial septal abnormalities in older patients with cryptogenic ischemic stroke or transient ischemic attack. Clinical Neurology and Neurosurgery, v. 110, p. $779-83,2008$.

GROGONO, J. et al. Simultaneous myocardial infarction and ischaemic stroke secondary to paradoxal emboli through a patent foramen oval. Clinical Medicine, v. 12, n. 4, 391-92, 2012.

GUERCINI, F. et al. Cryptogenic stroke: time to determine aetiology. Journal of Thrombosis and Haemostasis, v. 6, p. $549-54$. 
GUPTA, V. et al. Patent Foramen Ovale in a Large Population of Ischemic Stroke Patients: Diagnosis, Age Distribution, Gender, and Race. Echocardiography. A Jrnl. of CV Ultrasound \& Allied Tech, v. 25, n. 2, p. 217-27, 2008.

HOMMA, S.; Di TULLIO, M.R. Patent Foramen Ovale and Stroke. Journal of Cardiology, v. 56, p. 134-41, 2010.

JOHANSSON, M.C.; ERIKSSON, P.; DELLBORG, M. The significance of patent foramen ovale: A current review of associated conditions and treatment. International Journal of Cardiology, v. 134, p. 17-24, 2009.

KUTTY, S.; SENGUPTA, P.P.; KHANDHERIA, B.K. Patent foramen ovale: the known and the to be know. Journal of the American college of cardiology, v. 59, n. 19, 2012.

LANTZ, M.; SJOSTRAND, C.; KOSTULAS, K. Ischemic Stroke and Patent Foramen Ovale: Risk Factors and Genetic Profile. Journal of Stroke and Cerebrovascular Diseases, p. 1-5, 2012.

MATTLE, H.P.; MEIER, B.; NEDELTCHEV, K. Prevention of stroke in patients with patent foramen ovale. International Journal of Stroke, v. 5, p. 92-102, 2010.

ONORATO, E. et al. Patent foramen ovale closure: Pro and cons. Neurol Sci, v. 29, p.28-32, 2008.

OZDEMIR, A.Z. et al. Cryptogenic stroke and patent foramen ovale: clinical clues to paradoxal embolism. Journal of the Neurological Sciences, v. 275, p. 121-27, 2008.

PRISTIPINO, C. et al. Management of patients with patent foramen ovale and cryptogenic stroke: a collaborative, multidisciplinary, position paper. Catheterization and Cardiovascular Interventions, p. 1-33, 2012.

RIGATELLI, G.; CARDAIOLI, P.; CHINAGLIA, M. Asymptomatic Significant Patent Foramen Ovale: Giving Patent Foramen Ovale Management Back to the Cardiologist. Catheterization and Cardiovascular Interventions, v. 71, p. 573-76, 2008.

ROJAS, C. A. et al. Embryology and Developmental Defects of the Interatrial Septum. American Journal of Roentgenology, v. 195, n. 5, 2010.

RUNDEK, T. PFO in stroke: a direct association or coincidence? European Journal of Neurology, v. 15, p. 887-88, 2008.

TELMAN, G. et al. Size of PFO and amount microembolic signals in patients with ischaemic stroke or TIA. European Journal of Neurology, v.15, p. 969-72, 2008.

THALER, D. E.; SAVER, J. L. Cryptogenic stroke and patent foramen ovale. Current Opinion in Cardiology, v. 23, p. $537-44,2008$.

URIBE, M. H. J. Foramen ovale permeable y ataque criptogénico. Papel de la ecocardiografía y estado del arte. Revista Colombiana de Cardiología, v. 15, n. 4, 2008.

VIGNA, C. et al. Clinical and Brain Magnetic Resonance Imaging Follow-up After Percutaneous Closure of Patent Foramen Ovale in Patients With Cryptogenic Stroke. The American Journal of Cardiology, p. 1051-55, 2008.

WECHSLER, L. R. PFO and Stroke: What Are the Data? Cardiology in Review, v. 16, n. 1, 53-57, 2008.

WYNGAERT, F. et al. Absence of recurrent stroke after percutaneous closure of paten foramen ovale despite residual right-to-left cardiac shunt assessed by transcranial Doppler. Archives of Cardiovascular Disease, v. 101, p. 435-41. 